\title{
User-Driven Automation of Web Form Filling
}

\author{
Oscar Diaz, Itziar Otaduy, and Gorka Puente \\ Onekin Research Group, University of the Basque Country (UPV/EHU), \\ San Sebastián, Spain \\ \{oscar.diaz, itziar.otaduy, gorka. puente\}@ehu.es
}

\begin{abstract}
Form-intensive Web applications are common among institutions that collect bulks of data in a piecemeal fashion. European funding programs or income tax return illustrate these scenarios. Very often, most of this data is already digitalized in terms of documents, spreadsheets or databases. The task of manually filling Web forms out of these resources is not only cumbersome but also prone to typos. It does not benefit from the fact that the data is already in electronic format. Alternatively, externally-fed autofilling scripts can be programmed (e.g. using iMacros and Visual Basic) to code once, and enact many times. Unfortunately, this approach is programming intensive and fragile upon upgrades in either the website or the structure of the external source. This moves these tools away from users with scarce programming skills. We strive to empower these users by abstracting the way feeding solutions are realised. Since external sources tend to be structured, they offer the chance to be abstracted in terms of models. Autofilling scripts can then be generated as weavings between the external data model and the website model. We describe WebFeeder, a plugin for iMacros that introduces autofilling-script models as first-class artifacts in iMacros. The synthesis, enactment and maintenance of these script models are handled without leaving iMacros, minimizing users' cognitive load and involvement.
\end{abstract}

Keywords: autofilling, MDE, web forms, iMacros.

\section{Introduction}

Websites can be classified based on the quantity of data they request. If the requested data is mainly personal and limited, autofilling mechanisms are available to alleviate the tiresome task of periodically providing this information (card holder, visa number, etc.) [12. On the other side of the spectrum, some institutional websites request a large quantity of data. We qualified websites as "form-intensive" when they account for numerous web forms spread along several pages. In these scenarios, the manual approach is not only cumbersome but also prone to typos. Neither does it help the use of traditional autofilling mechanisms (e.g. Firefox autofill) where the filling data comes from previously filled forms but they do not benefit from external data sources. Indeed, it is very common in these scenarios for the required data to be already available within the organization. As an example, consider the application for R\&D projects.

F. Daniel, P. Dolog, and Q. Li (Eds.): ICWE 2013, LNCS 7977, pp. 171-185, 2013.

(C) Springer-Verlag Berlin Heidelberg 2013 
The schedule, personnel, budget, etc, are all data that might well be prepared in advance and stored as spreadsheets, documents or databases (e.g. if a wiki or a document management system is used). In addition, the same institution (e.g. a university) might present different projects to the same funding agency, which results in navigating the same website many times.

So far, only script-based approaches offer a solution. Scripts can be programmed using ad hoc languages (e.g. iMacros [3, Selenium [6]). These tools act like a record-replay device recording the interactions of the user during a session in terms of a script. This script can next be replayed at user's will. For external sources, the strategy rests on creating a program that consults the external source (e.g. a spreadsheet), assigning the returned values to variables, and next, enacting the script which was previously parametrized with these variables. This permits to tap into existing data sources while automatizing repetitive data entries.

For form-intensive websites, this approach offers a great potential. However, its benefits are hindered by:

- requiring an important upfront investment. Form-intensive websites necessarily lead to large scripts. This increases the chances of being affected by upgrades. Script development is programming intensive. The user has to code both the access to the external sources and the script using generalprogramming languages. In addition, this code shows external dependencies with the structure of both the external sources and the HTML pages. If upgrades are made on the structure on either the data source or the website, this code risks to fall apart,

- affordability. Form filling is a clerical work. Clerks manage the documents, spreadsheets and database applications that contain the data that will eventually feed the website. They know the site map, the possible flows for introducing the information as well as any directive concerning the feeding process. They are the domain experts as far as the feeding process is concerned. Unfortunately, clerks do not usually have programming skills, so current scripting tools are not affordable enough for this type of users.

This paper addresses the empowering of end users to create externallyfed autofilling scripts (hereafter just "scripts"). The approach rests on abstracting scripts from code to models. This stands for a decrease in human error and misunderstanding while improving efficiency and affordability. As a proof of concept, the paper describes how iMacros has been leveraged from managing script code to script models. Moving to the model realm improves iMacros affordability so that end users can now synthesise, run and maintain script models without requiring programming skills.

The paper starts by revising the related work and giving a brief about iMacros. Section 4 outlines the approach to abstract from script code to script models, which is later detailed throughout Sections 5, 6 and 7. Section 8 focuses on upgrades. Conclusions end the paper. 


\section{Related Work}

Autofilling is defined as a feature of a computer program that allows filling in forms without requiring user intervention. Aside from providing personal information, the autofilling feature can be useful in a large number of scenarios [14. Table 1 compares different solutions along four dimensions: mapping approach, data origin, user involvement and process concerns.

Mapping Approach. Autofilling implies a mapping between the data and the form fields. Three main solutions stand out to automatically infer this mapping. First, string matching based on HTML field attributes (e.g. name, label or id). This approach is illustrated by Google Toolbar [2] or Firefox Autofill Forms [1]. Second, semantic annotation e.g. using Microformats [4. Microformats can make a website machine-readable by adding special markup to mark recognizable data items (such as events, contact details or geographical locations). Firmenich et al. propose the use of Microformats to set up the mapping between HTML rendered elements and the data source [12]. A similar approach but using HTML5 features rather than Microformats is introduced in [13. Third, conceptual mapping. Unlike the previous techniques, now the mapping is achieved at the conceptual level. Form clues (e.g. id, label) are mapped to lexical words which are next compared with ontologies that contain synonyms and abbreviations, such as WordNet or DBpedia. Carbon [7] is a case in point. This application is "able to extract relevant metadata from the previously filled forms, semantically enrich it, and use it for aligning fields between web forms". Four, script-based mapping. Previous approaches trade accuracy for user involvement. That is, they reduce the engagement of the user at the expense of less precise results. If user collaboration is possible, the mapping can be recorded as a client-side script using tools such as iMacros [3] or Selenium [6]. These tools act like a record-replay device. Once recorded, the script can replay the interactions at user's will. This is useful if the user wants to fill out one form with different values (i.e. distinct replays) but it does not serve to automatically fill out new forms.

Data Origin. Feeding data can be obtained from previous feeding processes or existing documentation. The former is illustrated by Google Toolbar or Firefox

Table 1. Autofilling approaches

\begin{tabular}{|l|c|c|c|c|}
\hline & $\begin{array}{c}\text { Mapping } \\
\text { Approach }\end{array}$ & Data Origin & $\begin{array}{c}\text { User } \\
\text { Involv. }\end{array}$ & Process Concerns \\
\hline \hline $\begin{array}{l}\text { Firefox } \\
\text { Autofill }\end{array}$ & string matching & previous fillings & none & filling \\
\hline Carbon & $\begin{array}{c}\text { conceptual } \\
\text { mapping }\end{array}$ & previous fillings & none & filling \\
\hline iMacros & script-based & external sources & high & filling \& navigation \\
\hline WebFeeder & script-based & external sources & high & filling \& navigation \\
\hline
\end{tabular}


Autofill Forms. Alternatively, forms can be filled out from external sources. For example, Safari permits to tap into the Address Book [5] while CoScripter Tables [9] uses spreadsheets. In the commercial side, both iMacros and Selenium permit populating web forms from databases and text files.

User Involvement. This dimension admits three values based on the contribution of the user: (1) no-involvement: no additional effort is required from the user as data is automatically collected from the filling of other forms (e.g. Google Toolbar), (2) low involvement: the user provides an example that is later used to fill out similar forms, and (3), high involvement: the user facilitates a script that can be parametrized and replay with different values.

Process Concerns. The filling process can tackle different concerns. It can only focus on the filling, include data validation, or allow to be extended along different pages then, addressing page navigation as well. Most autofilling applications concentrate on form filling, though iMacros and Selenium also support navigation.

Table 1 frames our approach to related work. We focus on form-intensive websites. In this setting, feeding scripts from external sources offers an attractive solution to tap into existing resources within the organization. In addition, user involvement should be reduced on the search for making clerks self-sufficient. This rules out the manual programming of the scripts. Moreover, automatically obtaining the mapping using semantic closeness also turned out to be difficult when addressing a whole website. The main insight of this work is to tap into the information structure of the external sources to abstract and guide the mapping process so as to make it affordable to clerks. Therefore, the challenge is not so much about feasibility but affordability. Before delving into the details, we provide a brief on iMacros, the framework that underpins our approach.

\section{A Brief on iMacros}

Figure 1 depicts a web form for project application at the CDTI website, a Spanish funding body. Let us consider a scenario where: (1) the data has already been digitalized in terms of databases or spreadsheets, and (2) the organization (e.g. a university) applies for different projects, and hence, the very same forms need to be filled out over and over again. As in other software settings, repetitive tasks are worth being automatized through macros.

$i$ Macros is an extension for the main web browsers which adds record and replay functionality for a web session. The autofilling life cycle is conducted along two steps: (1) record the autofilling script as the user navigates throughout the site; and (2), replay the script at wish. Broadly, iMacros scripts are a sequence of navigation commands (e.g. the $U R L$ command opens a new webpage) and automated interactions on the current page (e.g. the TAG command performs some action on a web element). Autofilling wise, iMacros admits three strategies: (1) the data is provided at recording time as a script constant; (2) the data is provided at replaying time by prompting the user; and (3), the data is obtained 


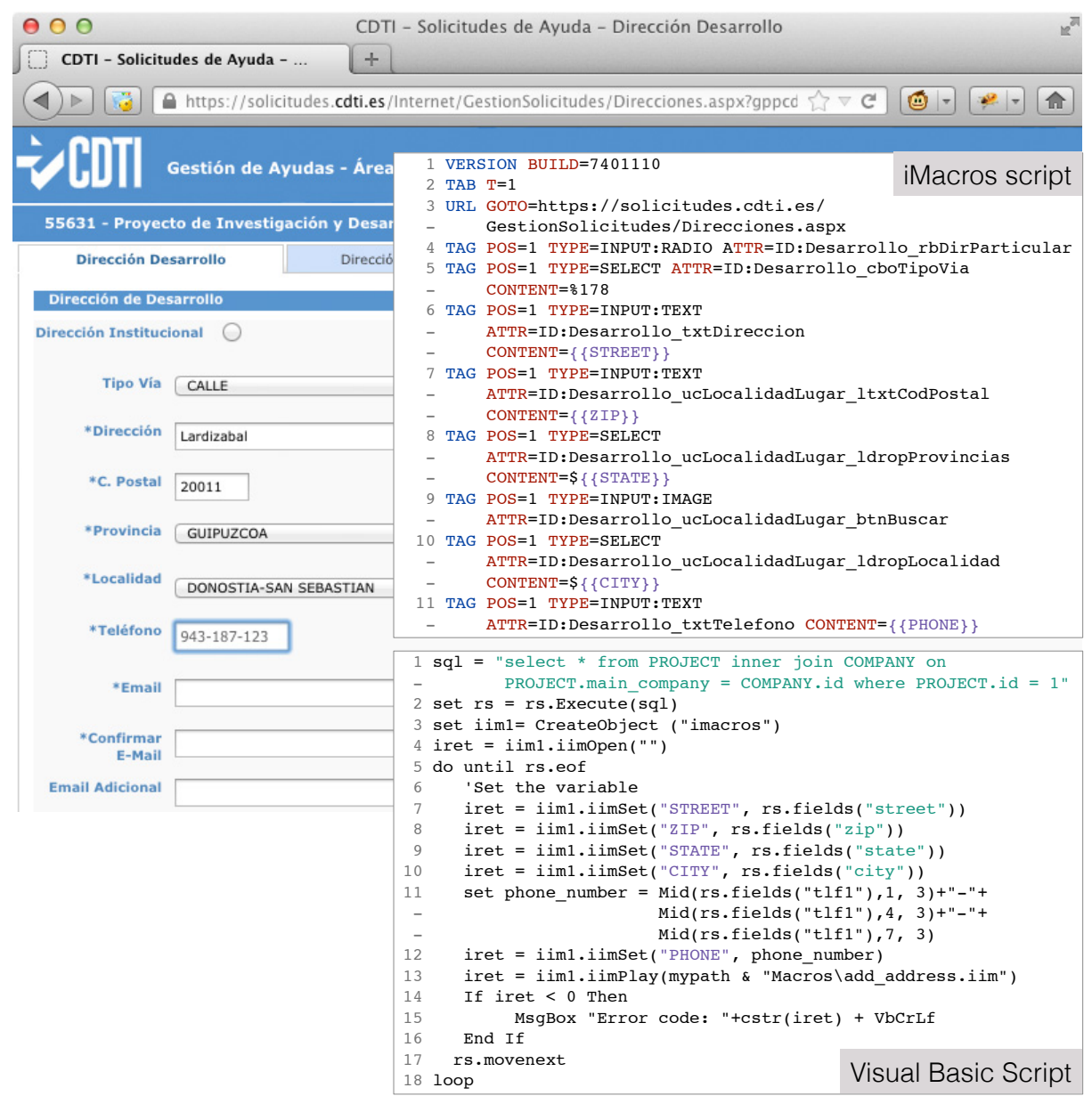

Fig. 1. CDTI form example. Parametrized iMacros script (top) and its VBS script configuration counterpart (bottom).

at replaying time by querying external sources. If data is provided at recording time, the autofilling follows the life cycle: "record > play". If data is to be obtained from external sources, the life cycle is enlarged with two additional steps: "record $>$ parametrize $>$ configure $>$ play". Parametrize basically means to turn values for data input into variables. Configuration implies to code a program that instantiates these variables.

Figure1 shows an example for the CDTI form sample. At the top, the iMacros script once parametrized as denoted by the expressions $\{\{$ variable $\}$. At the bottom, the configuration step which is realized through Visual Basic Script (VBS) code. Configuration mainly involves four concerns: querying the database (line 1), validating the data format (line 11), establishing database-to-script variable mappings (lines 7-10, 12) and enacting the iMacros script (line 13). By far, 


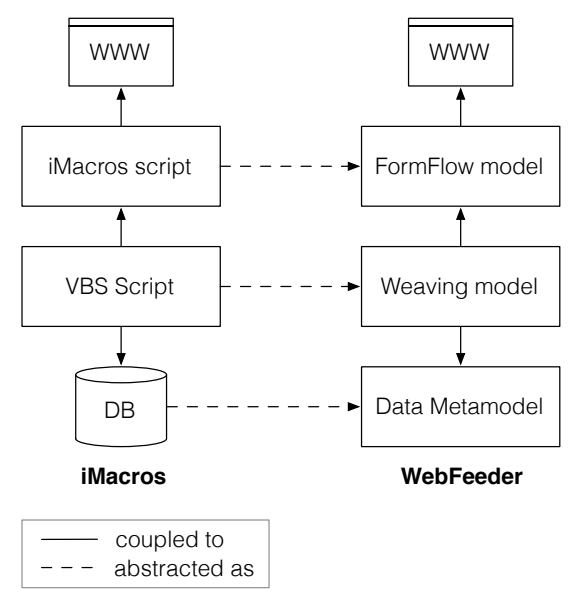

Fig. 2. From script coding to script modeling

establishing the mapping is the most complex task. Notice that parametrization and configuration are not supported by iMacros but handled externally (e.g. using a $V B S$ editor).

From a corporate perspective, the use of this solution for feeding form-intensive websites rises two issues. First, this solution might require an important upfront investment (e.g. the script for the CDTI case study took more than 20 hours to develop). This investment can be put in jeopardy if the structure of either the web pages or the external sources are upgraded. Second, affordability. Clerks are the domain experts as far as the feeding process is concerned. They know about the documents, spreadsheets and database applications that contain the data that will eventually feed the website. They also know about the site map, the possible flows for introducing the information as well as any directive concerning the feeding process. However, they cannot set the solution by themselves: $V B S$ is strange to them. Even a tiny change in the website (potentially breaking the script) makes them dependent on the availability of the always-busy computing department.

Configuration (i.e. the manual coding of the VBS script) is the Achilles' heel of this solution. The question is whether this code can be abstracted in terms of a model. This would bring modelware benefits to the realm of Web autofilling: simpler development, lower required skills, faster delivery, etc [8]. This grounds the development of WebFeeder.

\section{From iMacros to WebFeeder: From Coding to Modeling}

WebFeeder is a plugin for iMacros. iMacros is realized as a sidebar where artifacts (i.e. iMacros scripts) can be recorded, edited and played. We extended iMacros with a second type of artifact: feeders (see Figure 3). Feeders are abstractions of form filling scripts (i.e. script models). Feeders can be synthesised from iMacros scripts, and run, i.e. transformed from models into macros, 


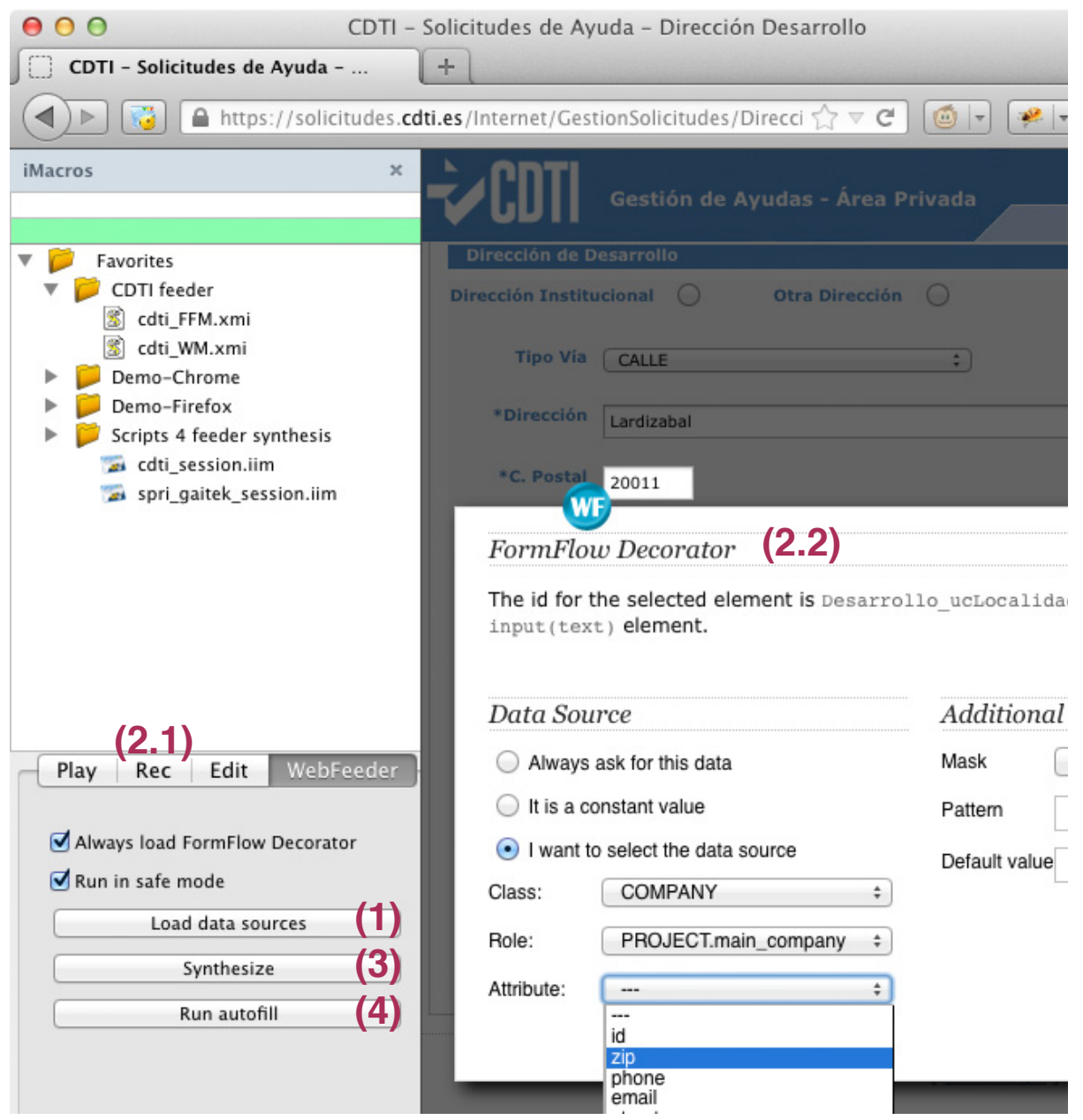

Fig. 3. WebFeeder extends iMacros to support the life cycle of feeders: record (2.1), weave (2.2), synthesise (3) and run (4)

and next, enacted. This round-trip from scripts to feeders yields the very same script if all data is constant and provided by the user at recording time. However, we extended iMacros with a configuration parameter: the external data sources (Figure 3(1)). This permits WebFeeder to obtain a rudimentary conceptual model from this external source before recording. At recording time (Figure 3. 2.1$)$, when an input field is detected, the user is prompted to set the mapping between the entry field and the conceptual model. Figure 3(2.2) shows this layered menu for the sample case. The C.Postal input field is mapped to the zip 


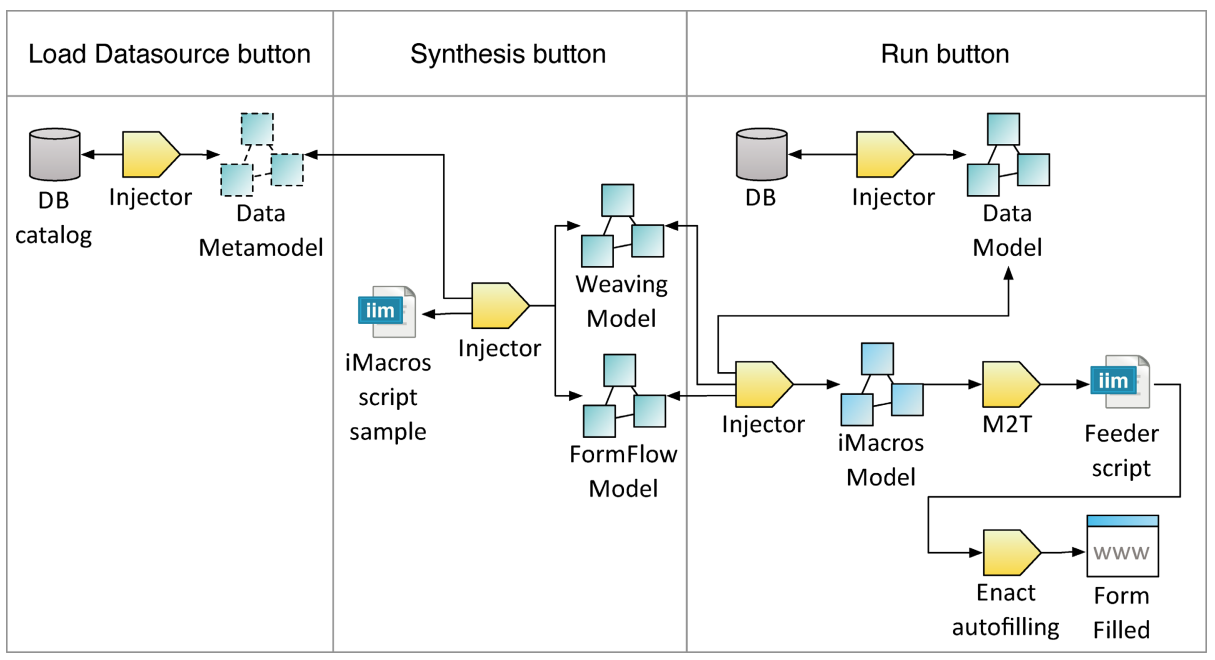

Fig. 4. Pushing the buttons in iMacros: the LoadDataSource button (Figure 3(1)), the Synthesis button (Figure 3(3)), and the Run button (Figure 3(4)).

attribute of the Company class when playing the role PROJECT.mainCompany. The important point to notice is that this mapping information is captured as part of the sample iMacros script being recorded. At synthesise time (Figure 3(3)), a feeder is obtained from the recorded macro and stored as part of the iMacros artifacts (e.g. the CDTI_feeder folder in Figure 3). At run time (Figure 3(4)), the feeder is transformed back into an iMacros script where mapping links are resolved during the transformation process so that the resulting script is a totally valid (i.e. totally instantiated) iMacros script. The whole process goes on without programming nor leaving the iMacros sidebar.

Implementation wise, three Ecore (meta)models are involved (see Figure 2(right)): (1) form filling scripts are abstracted in terms of FormFlow models, (2) the structure of the data source (e.g. the database schema) is captured as a Data metamodel, and (3), the VBS script is mainly expressed as a weaving model between a FormFlow model and a Data metamodel. The aforementioned feeders are realized as pairs (FormFlow model, Weaving model) (see the CDTI_feeder folder in Figure 3).

Feeders can be synthesised and run. Figure 4 depicts the processes triggered when pushing the namesake button in iMacros. During synthesis, injectors are used to obtain the FormFlow model (i.e. the platform-independent model (PIM)) and the Weaving model out of the sample iMacros script. At run time, the feeder is enacted, i.e, ( $i$ ) references to external sources are resolved, (ii) an iMacros model (i.e. the platform-specific model (PSM)) is generated merging the information contained into the FormFlow, the Weaving and the Data models, (iii) this iMacros model is transformed into an iMacros script, and (iv) this script is run. Next sections introduce the main models that comprise the WebFeeder system and their extraction processes. 


\section{$5 \quad$ Abstracting the External Sources}

The Data Metamodel. It stands for the elements and structure of the external source. If a database then, the Data metamodel captures the database schema as represented in the database catalogue. If a spreadsheet then, the Data metamodel denotes the tabular structure where data is ordered along different sheets and columns.

Injection. No matter the data source, the challenge is twofold. First, we need "a metamodel injector" that automatically obtains the Data metamodel out of the structure of the data source (e.g. the database schema, the spreadsheet file structure). Second, we require a "model injector" which harvests models out of a data source according to a given metamodel.

In a previous work [11, we studied model injection for databases, providing a language, Schemol, for defining database-to-model injectors. Schemol also permits to perform an automatic extraction of a metamodel based on a database schema. This feature, called bootstrap, transforms tables into metaclasses, columns into attributes, and foreign keys into references between metaclasses. This work has been extended to permit data sources other than databases. The approach is based on defining appropriate "drivers" that permit to conceptualize spreadsheets as databases, where sheets are the tables counterparts. The Schemol engine is the same but the driver changes. So far, drivers are available for Excel spreadsheets. Figure 5(right) shows the Data metamodel automatically obtained by Schemol for our sample database (Figure 5(left)). Each project has a main company, a set of participating companies, a manager and a set of users. Meanwhile, each employee is associated with the company he works for. Both companies and employees can be associated with several projects.

\section{Abstracting the Form Filling Process}

The FormFlow Metamodel. A FormFlow model abstracts the process of filling in a specific web form. This process abstracts a valid sequence of user interactions with the aim of form filling. The model includes: $(i)$ the elements that compose the web form (e.g., type of inputs, ids), (ii) the order of the interactions (e.g., which input comes after another, when do we have to click a button, etc.) and (iii) the existence of loops (i.e. a set of pages that can be filled more than once during the same process, e.g. adding many users to a project).

A FormFlow model abstracts a filling process through a Session class (see Figure (6). A session is a sequence of PageVisits which in turn, are conceived as sequences of Interactions. Interactions can be AtomicInteractions or CompoundInteractions. Atomic interactions are classified as mouse Click or data Input. Clicks act upon Hyperlinks or Buttons, and they are usually used for page navigation. Input elements require the user to introduce data. On the other hand, a compound interaction stands for a meaningful unit of interactions (e.g. an HTML form element). Loops can be defined when many similar elements need to be introduced through the same section of the web form (e.g. adding 


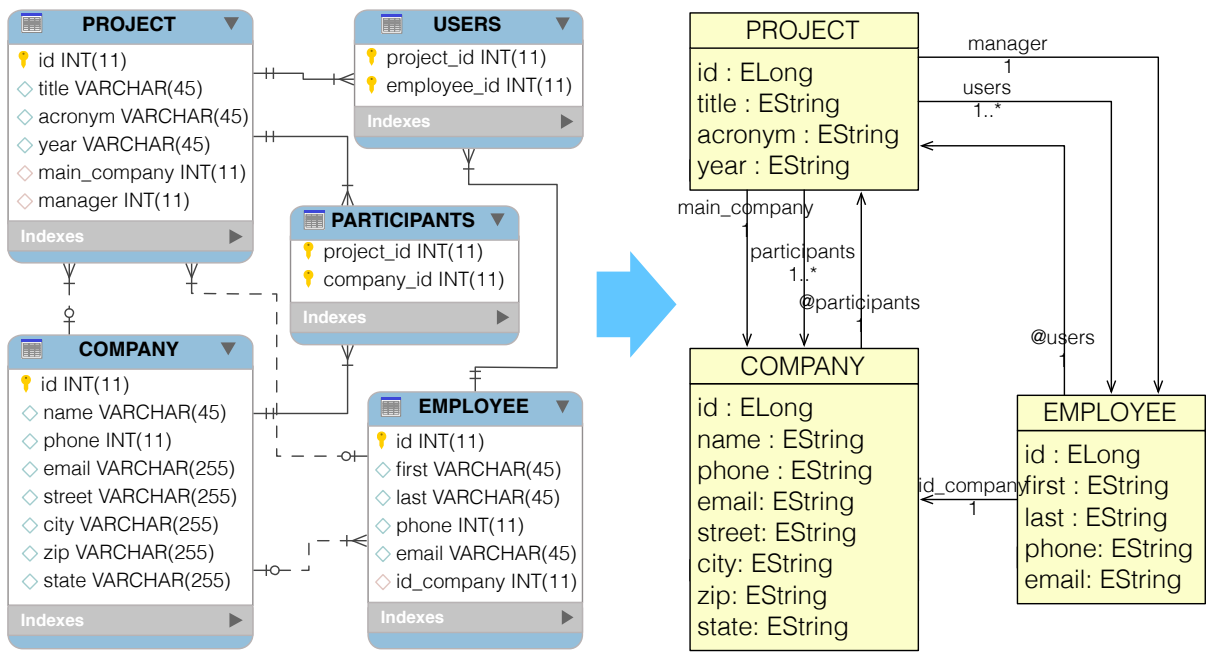

Fig. 5. Harvesting the Data metamodel out of database catalogues

users to a project). Each loop contains a reference to its trigger interaction (i.e. the button to access the first form in the loop) and the set of involved pageVisits.

Injection. FormFlow models can be automatically obtained from iMacros scripts. This process goes through the recorded macro and for each action, creates the corresponding FormFlow model element. For each URL or TAG command performed on a button or hyperlink, a Click element is created. On the other hand, when a TAG command is performed on an HTML input field, an Input element is generated. If the system detects that one command has been enacted in a different page than the previous one, a new PageVisit is generated. Within each visited page, for each different HTML form element whose fields have been enacted a CompoundInteraction is created. Besides, if the transformation detects that a concrete set of pages have been filled out more than once, it automatically creates the corresponding Loop element. Figure 7 depicts the FormFlow model obtained from the iMacros script in Figure 1.

\section{$7 \quad$ Abstracting the Mapping}

The Weaving Metamodel. A mapping sets a feed relationship from attributes in the Data metamodel to input fields in the FormFlow model. This mapping is captured as "link" elements along an AMW model [10]. A link states a nexus (in this case, a feed relationship) that indicates which data is to feed what input field.

Injection. Weaving data is collected as part of the recording process. That is, we prompt the user for the weaving information when iMacros encounters an input field. This is achieved through the FormFlowDecorator (see Figure $3(2.2)$ ). When 


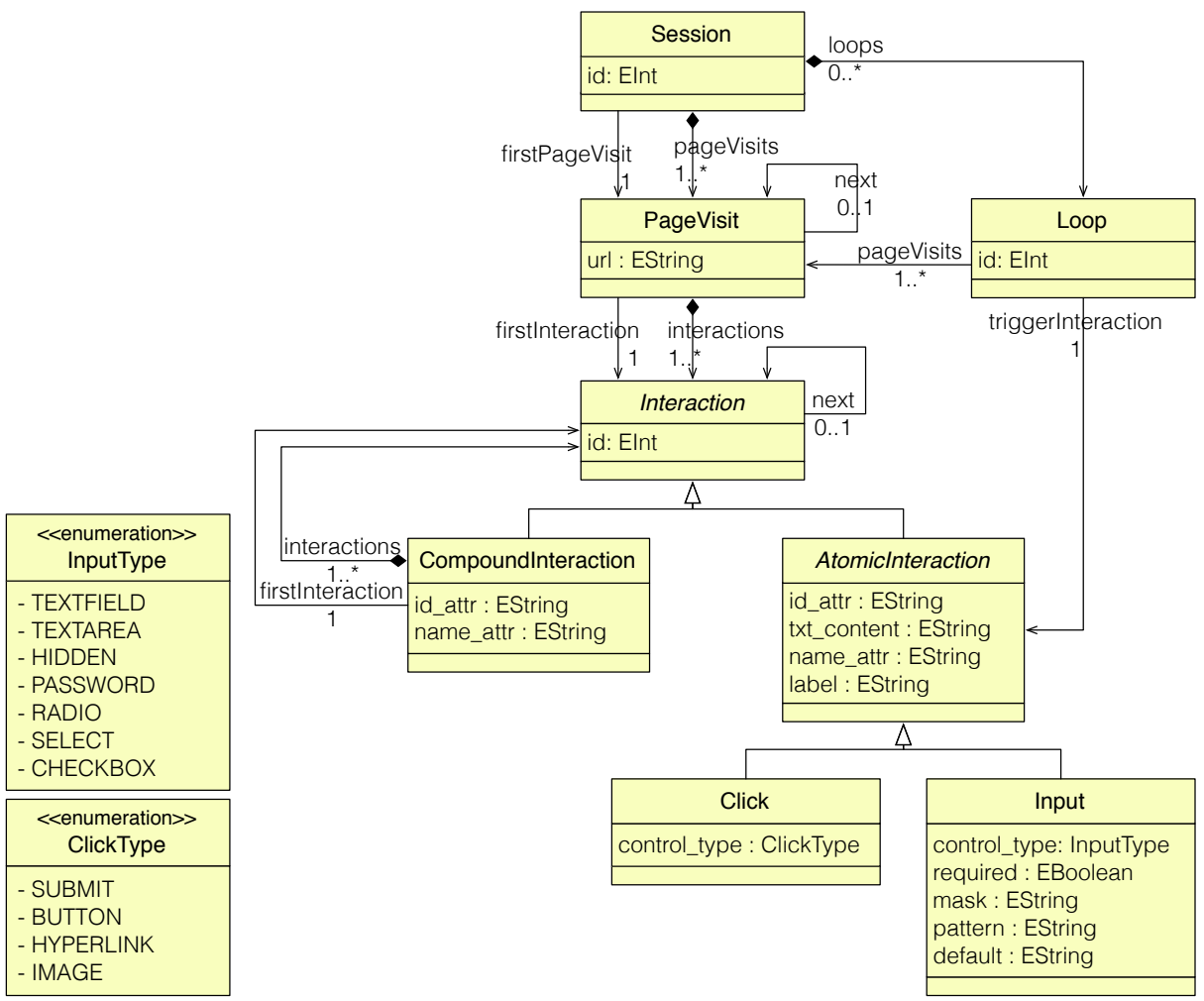

Fig. 6. The FormFlow metamodel

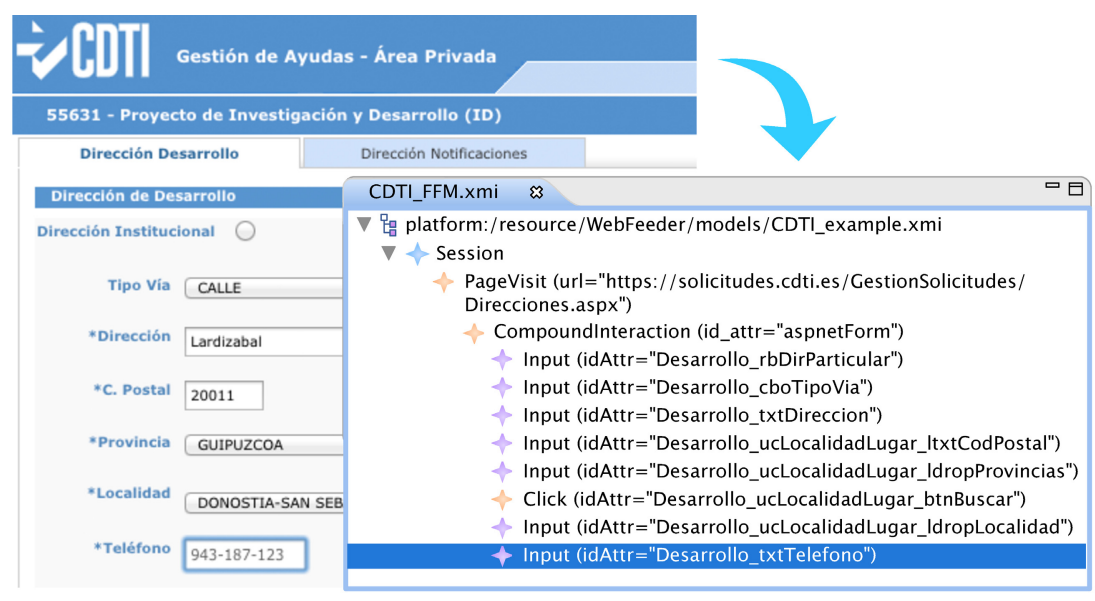

Fig. 7. Harvesting the FormFlow model out of iMacros scripts 
an input field is detected, the FormFlowDecorator pops up to collect the other participant in the link relationship: the attribute of the Data metamodel. In the example, the input field C.Postal is detected in a web form. This makes the decorator pop up, requesting its Data metamodel counterpart. In this way, the iMacros script is leveraged with weaving data as part of the recording process. In addition, the user can also provide the mask, the pattern and a default value for the input field at hand. Similar to the previous cases, an injector is defined to extract the Weaving model out of the enriched iMacros script. No additional user intervention is required.

\section{Facing Upgrades}

Section 3 characterises current solutions as being programming intensive and fragile. WebFeeder moves this endeavour from the programming realm to the modeling realm, and in so doing, reduces the effort and the skills required to obtain a solution. However, models (i.e. feeders) are still fragile. That is, upgrades on either the website or the structure of the data source can make the feeder break apart. This section addresses this issue.

Table 2. A classification of upgrades for websites and database schema. Frequency is based on anecdotal evidences from the test case.

\begin{tabular}{|c|c|c|}
\hline Change & Frequency & Contingency action \\
\hline \hline Create table & low & None \\
\hline Drop table & low & Update Weaving model \\
\hline Add column & high & None \\
\hline Drop column & high & Update Weaving model \\
\hline \hline New Page & low & Regenerate the feeder from start \\
\hline Delete Page & low & Regenerate the feeder from start \\
\hline New Form & low & Update FormFlow model \\
\hline Delete Form & low & Update FormFlow model \\
\hline New Field & high & Update FormFlow model \\
\hline Delete Field & high & Update FormFlow model \\
\hline
\end{tabular}

Table 2 typifies some of the possible changes. Upgrades can be handled using corrective or preventive actions. A corrective action deals with an upgrade that has occurred, and a preventive action addresses the potential for an upgrade to occur. We opted for a preventive strategy for tackling upgrades. That is, before running the feeder, we first check whether an upgrade occurred. If so, the user is prompted so as to reestablish the consistency between the feeder and the external dependencies (i.e. the website or the database schema). To this end, we introduce the "safe mode" for running feeders. Microsoft Windows' safe mode is a boot method that facilitates to diagnose problems. Likewise, when upgrades are expected, feeders can be run in "safe mode". Compared with the 
normal execution, this mode introduces two main differences as for the feederto-iMacros transformation, namely:

- the transformation is not enacted as a single shot but it processes one PageVisit at a time. This introduces a kind of "lazy evaluation" where the transformation of pageVisit elements and the enactment of the resulting iMacros scripts are intermingled: transform(pageVisit_1, scriptOutput_1), enact(scriptOutput_1), transform(pageVisit_2, scriptOutput_2), enact(scriptOutput_2), etc. The rationales are twofold. First, this permits to phrase upgrade detection in terms of model differences between the existing pageVisit model and the current pageVisit model as extracted from the current page . Second, upgrade resolution is also handled at the page level, hence facilitating the intervention of the user at the time and at the place where the mismatch is detected (see next point).

- the transformation is leveraged with "caution clauses". Two types of caution clauses are introduced to handle each type of upgrades. For upgrades on web pages, a caution clause is introduced before the generation of the corresponding iMacros script. On loading, the clause obtains the FormFlow model only for the page at hand: the current "pageVisit" model. This model is compared for the namesake page in the existing FormFlow model: the stored "pageVisit" model. If mismatches, the FormFlowDecorator pops up for the user to restore the consistency. Second, for upgrades on external sources, a caution clause is introduced after the update of each pageVisit. This clause checks the mapping established on the Weaving model with the existing Data metamodel elements. If a reference does not match any Data metamodel element, the user is prompted to restore the consistency by providing a new mapping. Once these models are updated, the autofilling for the current page is enacted.

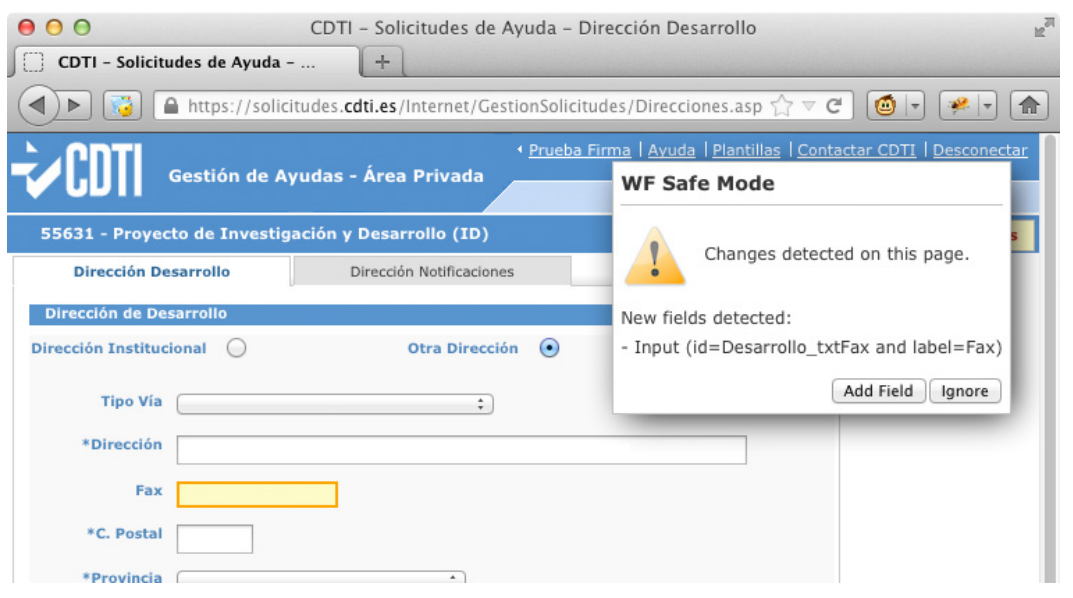

Fig. 8. Upgrading the sample form with a new field 
Figure 8 illustrates the case of upgrading the sample form with a new field. WebFeeder detects a mismatch between the current FormFlow model and the pageVisit model as extracted from the current page. The execution stops and the user is prompted to provide a data weaving for the new field (if appropriate). Next, the execution resumes.

The bottom line is that feeder co-evolution is handled using the very same mechanisms that those of feeder construction, hence minimizing the cognitive burdens. Clerks can cope with (small) upgrades by themselves without turning to technical staff. Disruptive upgrades like introducing new tables or adding or deleting pages, require re-generating the feeder from scratch.

\section{Conclusions}

We address the feeding of form-intensive websites from external sources. Current solutions such as iMacros are characterized as programming intensive and fragile, hence, moving these tools away from their more likely audience: clerks. We strive to empower back clerks by abstracting the way at which feeding solutions are realized. The approach abstracts the development effort from the coding of iMacros scripts to the conception of models (i.e. feeders) from which these scripts are generated. In addition, feeder co-evolution (i.e. propagating website/data structure upgrades to the feeder) is handled using the very same mechanisms that those of feeder construction, hence minimizing the cognitive burdens. Clerks can cope with (small) upgrades by themselves without resorting to technical staff. The approach is realized through WebFeeder, a plugin for iMacros. WebFeeder introduces script models (i.e. feeders) as first-class artifacts in iMacros. Feeder synthesis, enactment and maintenance is handled without leaving iMacros. Next follow-on includes to conduct studies on the robustness and affordability of WebFeeder. A detailed account of the typology of website upgrades and their likelihood, will serve to better assess WebFeeder robustness. So far, only one clerk has participated in the design of WebFeeder. A more ample feedback is required.

Acknowledgments. Thanks are due to Inmaculada Cacho for her evaluation of WebFeeder. This work is co-supported by the Spanish Ministry of Education, and the European Social Fund under contract TIN2011-23839, and the Ministerio de Industria, Turismo y Comercio under contract TSI-020500-2010-206. Otaduy enjoys a doctoral grant from the Basque Government under the "Researchers Training Program".

\section{References}

1. Firefox autofill forms plugin. Online, http://autofillforms.mozdev.org (last accessed February 19, 2013)

2. Google toolbar. Online, http://toolbar.google.com/ (last accessed February 19, 2013) 
3. iMacros. Online, http://www.iopus.com/iMacros/ (last accessed February 19, 2013)

4. Microformats. Online, http://microformats.org// (last accessed February 19, 2013)

5. Safari - autofill. Online http://www.apple.com/safari/ (last accessed February 19, 2013)

6. Selenium plugin. Online, http://docs.seleniumhq.org (last accessed February 19, 2013)

7. Araujo, S., Gao, Q., Leonardi, E., Houben, G.-J.: Carbon: Domain-Independent Automatic Web Form Filling. In: Benatallah, B., Casati, F., Kappel, G., Rossi, G. (eds.) ICWE 2010. LNCS, vol. 6189, pp. 292-306. Springer, Heidelberg (2010)

8. Brambilla, M., Cabot, J., Wimmer, M.: Model-Driven Software Engineering in Practice. Synthesis Lectures on Software Engineering. Morgan \& Claypool Publishers (2012)

9. Cypher, A.: Automating Data Entry for End Users. In: 2012 IEEE Symposium on Visual Languages and Human-Centric Computing (VL/HCC), pp. 23-30 (2012)

10. Fabro, M.D.D., Bézivin, J., Valduriez, P.: Weaving Models with the Eclipse AMW Plugin. In: Eclipse Modeling Symposium, Eclipse Summit Europe, Citeseer, vol. 2006 (2006)

11. Díaz, O., Puente, G., Izquierdo, J.L.C., Molina, J.G.: Harvesting Models from Web 2.0 Databases. Software \& Systems Modeling 12(1), 15-34 (2013)

12. Firmenich, S., Gaits, V., Gordillo, S., Rossi, G., Winckler, M.: Supporting Users Tasks with Personal Information Management and Web Forms Augmentation. In: Brambilla, M., Tokuda, T., Tolksdorf, R. (eds.) ICWE 2012. LNCS, vol. 7387, pp. 268-282. Springer, Heidelberg (2012)

13. Heinrich, M., Gaedke, M.: WebSoDa: A Tailored Data Binding Framework for Web Programmers Leveraging the WebSocket Protocol and HTML5 Microdata. In: Auer, S., Díaz, O., Papadopoulos, G.A. (eds.) ICWE 2011. LNCS, vol. 6757, pp. 387-390. Springer, Heidelberg (2011)

14. Scaffidi, C., Cypher, A., Elbaum, S., Koesnandar, A., Myers, B.: Using Scenariobased Requirements to Direct Research on Web Macro Tools. Journal of Visual Languages \& Computing 19(4), 485-498 (2008) 